\title{
Numerical Simulation of Aerodynamic Performance of Off-grid Small Vertical Axis Wind Turbine
}

\author{
Qiuyun Mo ${ }^{1,2}$, Jiabei Yin ${ }^{2, *}$, Lin $\mathrm{Chen}^{2}$, Weihao Liu' ${ }^{2}$ Li Jiang ${ }^{2}$, and Zhiqiang Liao ${ }^{2}$ \\ ${ }^{1}$ Guangxi Experiment Center of Information Science, Guilin University of Electronic Technology, Guilin, 541004, China \\ ${ }^{2}$ Department of Electrical and Mechanical Engineering, Guilin University of Electronic Technology, Guilin, 541004, China
}

\begin{abstract}
In this paper, a 2D off-grid small compact model of vertical axis wind turbine was established. The sliding grid technology, the RNG turbulence model and the Coupld algorithm was applied to simulate the unsteady value of the model's aerodynamic performance. Through the analysis on the flow field at difference moments, the rules about velocity fields, vortices distributions and the wind turbine's total torque were obtained. The results show that: the speed around wind turbine blades have obvious gradient, and the velocity distribution at different times show large differences in the computional domain. In the rotating domain vorticity is large. With away from the rotation domain, vorticity reduced quickly. In the process of rotating for vertical axis wind turbine, the wind turbine's total torque showed alternating positive and negative changes.
\end{abstract}

\section{Introduction}

With the depletion of non-renewable energy sources and the continuous deterioration of the environment, the use of renewable energy sources has been valued. Wind energy is a clean, renewable energy source. Wind power generation is the most renewable energy generation method with the most extensive development conditions and commercial development prospects. The key to wind energy capture by wind turbines lies in the aerodynamic performance of wind turbines. The main research methods for the aerodynamic performance of wind turbines include model method, numerical calculation method and wind tunnel test method. For wind tunnel tests are limited by experimental conditions and technical levels, also the cost is high and the cycle is long. Therefore, the model method plays a major role in the initial stage of the wind turbine. The main theoretical models for vertical axis fans foreign and domestic are: momentum model, eddy current model and cascade model, of which the first two are more commonly used. Although the numerical calculation method began to appear in recent years, it has developed rapidly and has now become a major design method.

This paper was based on the finite volume method of Computional Fluid Dynamics (CFD). The sliding grid technology and the two-dimensional unsteady numerical simulation of small vertical axis wind turbine were used to research flow characteristics and aerodynamic performance. It provided a certain theoretical basis for the design of vertical axis wind turbine.

\section{Numerical methodology}

\subsection{Control equations}

Fluid flow should follow the law of physical conservation, which is the law of conservation of mass, momentum, and energy. For two-dimensional incompressible flow, two-dimensional incompressible $\mathrm{N}-\mathrm{S}$ equations and continuity equations can be established based on the principles of mass, momentum, and energy conservation and continuity equations.

$$
\begin{gathered}
\frac{\partial(\rho \mathrm{u})}{\partial \mathrm{t}}+\mathrm{u} \frac{\partial(\rho \mathrm{u})}{\partial \mathrm{t}}+\mathrm{v} \frac{\partial(\rho \mathrm{u})}{\partial \mathrm{y}}=-\frac{\partial \mathrm{p}}{\partial \mathrm{x}}+\mu\left(\frac{\partial^{2} \mathrm{u}}{\partial \mathrm{x}^{2}}+\frac{\partial^{2} \mathrm{u}}{\partial \mathrm{y}^{2}}\right) \\
\frac{\partial(\rho \mathrm{u})}{\partial \mathrm{t}}+\mathrm{u} \frac{\partial(\rho \mathrm{v})}{\partial \mathrm{t}}+\mathrm{v} \frac{\partial(\rho \mathrm{v})}{\partial \mathrm{y}}=-\frac{\partial \mathrm{p}}{\partial \mathrm{x}}+\mu\left(\frac{\partial^{2} \mathrm{v}}{\partial \mathrm{x}^{2}}+\frac{\partial^{2} \mathrm{v}}{\partial \mathrm{y}^{2}}\right) \\
\frac{\partial \mathrm{u}}{\partial \mathrm{x}}+\frac{\partial \mathrm{v}}{\partial \mathrm{y}}=0
\end{gathered}
$$

\subsection{Turbulence model}

In comparison with the standard k- $\varepsilon$ model, the RNG k- $\varepsilon$ model modifies the turbulence model, taking the effects of rotating and rotating flows into consideration, and adding an $\mathrm{E}_{\mathrm{ij}}$ to the $\varepsilon$ equation to reflect the dominant time-averaged strain rate. It makes the generation term in the RNG $\mathrm{k}-\varepsilon$ model only related to the flow conditions and a function of the space coordinates; whereas the standard k- $\varepsilon$ model can be used to apply negative normal stress when the average strain rate is particularly large. The external flow field of the vertical axis wind turbine is unsteady flow, and the rotation of the wind turbine produces a strong rotation effect. The selection of the RNG k- $\varepsilon$ turbulence model will be more appropriate.

$\frac{\partial(\rho \mathrm{k})}{\partial \mathrm{t}}+\frac{\partial\left(\rho \mathrm{ku}_{\mathrm{i}}\right)}{\partial \mathrm{x}_{\mathrm{i}}}=\frac{\partial}{\partial \mathrm{x}_{\mathrm{i}}}\left[\alpha_{\mathrm{k}} \mu_{\mathrm{eff}} \frac{\partial \mathrm{k}}{\partial \mathrm{x}_{\mathrm{i}}}\right]+\mathrm{G}_{\mathrm{k}}+\rho \varepsilon$ 


$$
\frac{\partial(\rho \varepsilon)}{\partial \mathrm{t}}+\frac{\partial\left(\rho \varepsilon \mathrm{u}_{\mathrm{i}}\right)}{\partial \mathrm{x}_{\mathrm{i}}}=\frac{\partial}{\partial \mathrm{x}_{\mathrm{i}}}\left[\alpha_{\varepsilon} \mu_{\mathrm{eff}} \frac{\partial \mathrm{k}}{\partial \mathrm{x}_{\mathrm{i}}}\right]+\frac{\mathrm{C}_{1 \varepsilon}^{*}}{\mathrm{k}} \mathrm{G}_{\mathrm{k}}-\mathrm{G}_{2 \varepsilon} \rho \frac{\varepsilon^{2}}{\mathrm{k}}
$$

\subsection{Sliding grid technology}

Both MRF and MPF ignore the unsteady interactions on both sides of the interface. In some engineering applications, when the interactions on both sides of the interface are not negligible, MRF and MPF cannot be used to solve the problem, using a slip grid for transient solution instead. These interactions are strong when the stator and the rotor interact with potentials, wakes and vibrations. The sliding grid is usually used for solving calculations. However, it must ensure that the geometry on both sides of the interface is consistent and the mesh interface must not be normal movement. As the wind turbine rotates, the position, relative speed and angle of attack of the blades periodically changed with time. Therefore, the unsteady flow of the two-dimensional flow field was calculated, and the sliding grid model was used to simulate the rotation of the wind wheel. The law of flow field changed overtime.

\section{Off-grid small vertical axis wind turbine CFD model}

\subsection{VAWT geometrical and operational characteristics}

A 3-blade H-type low-solidity VAWT equipped with NACA0015 airfoils and swept area of $1 \mathrm{~m}^{2}$ was simulated in $2 \mathrm{D}$ at a constant of 2.2. The turbine rotational velocity is $44 \mathrm{rad} / \mathrm{s}$ and the free-stream velocity was $10 \mathrm{~m} / \mathrm{s}$. The geometrical and operational characteristics of the VAWT were presented in Table1.

Table 1. Geometrical and operational characteristics of the VAWT.

\begin{tabular}{|c|c|}
\hline Characteristics & Turbine \\
\hline Number of blade & 3 \\
\hline Diameter(m) & 1 \\
\hline Swept area(m $\left.{ }^{2}\right)$ & 1 \\
\hline Airfoil & NACA0015 \\
\hline Airfoil chord(m) & 0.2 \\
\hline Tip speed ratio & 2.2 \\
\hline Free-stream velocity $(\mathrm{m} / \mathrm{s})$ & 10 \\
\hline Rotational speed(rad/s) & 44 \\
\hline
\end{tabular}

\subsection{Computional domain and grid}

For the rotor geometry, only the turbine's blades were taken into account, neglecting the presence of both the supporting struts and the shaft. The simulation domain were divided into two sub-domains, a circular inner zone containing the turbine rotating with the same angular velocity of the rotor and a rectangular fixed outer zone determining the overall domain extent. The inlet, lateral boundaries and the outlet were placed at a distance from the rotational axis of the turbine of 10 diameters. The diameter of the rotating region was 1.75 diameters. These were shown in Fig.1. Encrypt the grid near the blades in a partitioned and differently-divided way, and determine the total number of nodes in the calculation area by trial calculation to be 316677 . The total number of elements is 315293. Sliding grid was used to handle coupling between rotating and non-rotating region. The incompressible unsteady Reynolds-averaged Navier-Stokes (URANS) equations were solved using the commercial CFD software package ANSYS Fluent 18.1. The boundary conditions at the inlet, outlet, side faces and walls were uniform velocity, zero surface-averaged gauge pressure, symmetry and no slip, respectively.

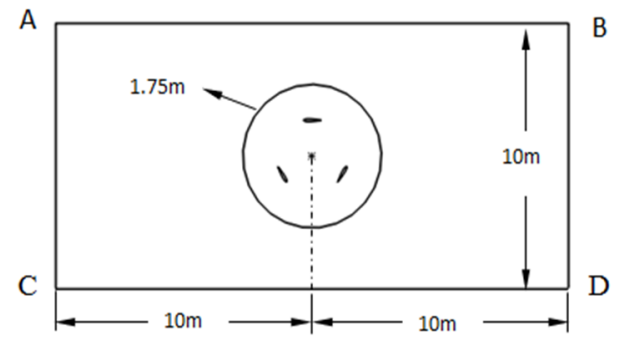

Fig. 1. Computational domain.

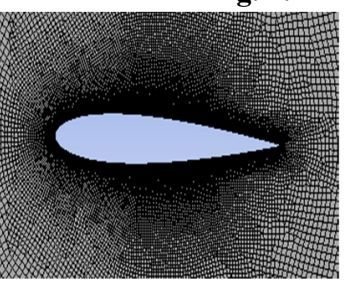

(a)

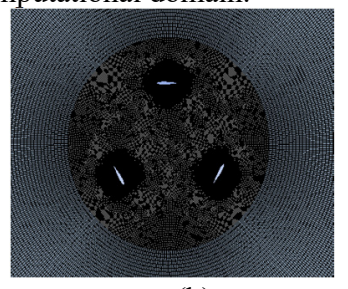

(b)
Fig. 2. Computional grid: (a) Near the airfoil; (b) Near the rotating core.

\section{Numerical simulation analysis}

\subsection{Velocity distribution}

The calculation time step was $0.001 \mathrm{~s}$, and the flow field was analyzed after intercepting the flow field for a stable time of $2.4 \mathrm{~s}, 2.5 \mathrm{~s}, 2.6 \mathrm{~s}$ and $2.7 \mathrm{~s}$. The corresponding angles are $6050^{\circ}, 3602^{\circ}, 6554^{\circ}$ and $6806^{\circ}$. As shown in Figure.3, the upstream flow velocity in the calculation area was close to the inlet flow velocity, and the velocity gradient around the blade was significant. The velocity in the downstream part of the calculation area was greater than the velocity of the free air flow, which reflected the influence of the rotation of the wind turbine on the downstream area. As it was far away from the rotating area, the flow velocity gradually approaches the wind speed of the free air flow, mainly due to the convection effect between the wake flow and free air flow. From Figure.3, it could also be seen that there was a large difference in the speed distribution of the calculation area at different times. This reflected the influence of the rotation of the wind turbine to different locations on the structure of the flow field. 


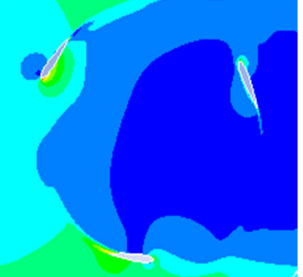

(a)

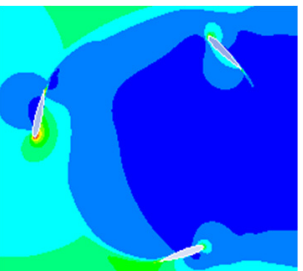

(c)

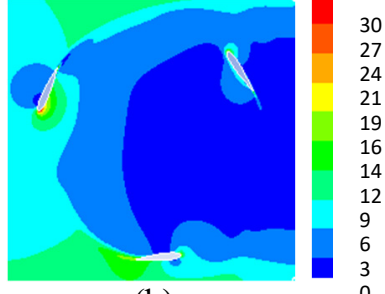

(b) Velocity $(\mathrm{m} / \mathrm{s})$

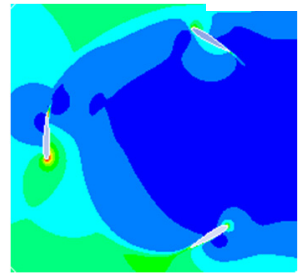

(d)
Fig. 3. Velocity distributions around the blades: (a) $t=2.4 s$; (b) $\mathrm{t}=2.5 \mathrm{~s}$; (c) $\mathrm{t}=2.6 \mathrm{~s}$; (d) $\mathrm{t}=2.7 \mathrm{~s}$.

\subsection{Flow field structure analysis}

The vorticity distribution maps of the flow field at $2.4 \mathrm{~s}$, $2.5 \mathrm{~s}, 2.6 \mathrm{~s}$ and $2.7 \mathrm{~s}$ were shown in Fig.4. When the blades were in a state of small angle of attack, the flow around the blades was an adherent flow. As the angle of attack increases and was greater than the critical angle, the separation flow occurs first on the upper surface of the trailing edge of the blade. As the angle of attack increases further, the separation point moves toward the leading edge of the blade and the separation zone gradually increases. When the angle of attack was greater than a certain angle, the separation of the vortex occurs on the upper surface of the blade, and it alternately fell off to the downstream. The vortex was rapidly dissipated in the flow field after it was detached from the wall surface of the blade, but it still had some interference with the downstream blade. In general, the vorticity was relatively large and the amplitude of change was very sharp in the area near the wall of the blade. The vorticity was greater in the rotating area of the vertical axis wind turbine and vortices were also distributed in the downstream of the calculated area. The vorticity value decreases rapidly as it was far away from the rotating area, which indicates that the vertical axis wind turbine had a little influence on the downstream flow field during operation.

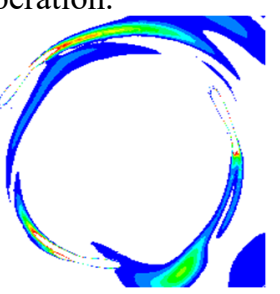

(a)

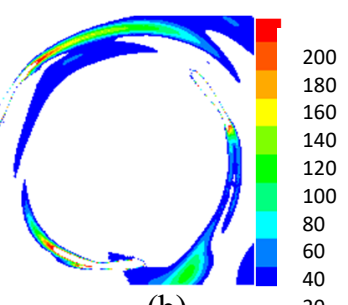

(b) $\operatorname{vorticity}(1 / \mathrm{s})$

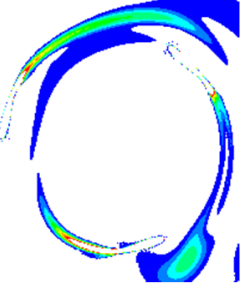

(c)

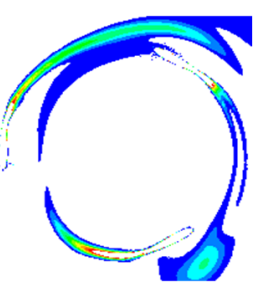

(d)
Fig. 4. Vorticity distributions around the blades: (a) $t=2.4 s$; (b) $\mathrm{t}=2.5 \mathrm{~s}$; (c) $\mathrm{t}=2.6 \mathrm{~s} ;$ (d) $\mathrm{t}=2.7 \mathrm{~s}$.

\subsection{Wind wheel moment characteristics}

Figure.5 showed that the wind turbine torque characteristics during the rotation of the wind turbine. The total moment of the calculated model had an obvious periodicity, starting from $0.9 \mathrm{~s}$, which indicated that the flow field had fully developed at this time. When the rotation speed is $44 \mathrm{rad} / \mathrm{s}$, the total moment of the calculation model changed alternately positively and negatively. This was mainly caused by the continuous change of the angle of attack of the blade during the rotation of the wind turbine. Although the total torque of the calculation model alternated positively and negatively during the rotation, the work done to propel the wind turbine during the rotation was greater than the work done to prevent the torque of the wind turbine from turning.

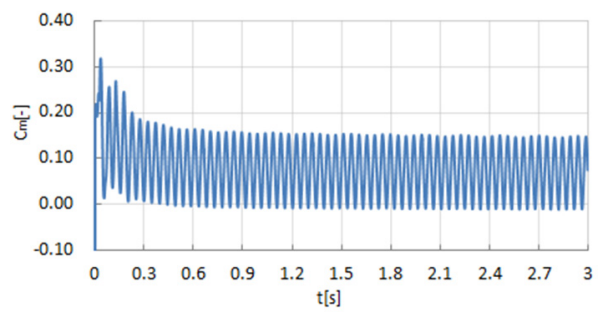

Fig. 5. Moment coefficient of NACA0015

\section{Conclusions}

By establishing a VAWT model and using fluent software, the finite volume method was used to simulate the flow field around the VAWT. The flow field characteristics were obtained and analyzed. The calculation results show that: (1) The velocity gradient around the blades of the vertical axis wind turbine was obvious, and the flow velocity gradually approached the wind velocity of the free air flow as it moved away from the rotating region. The velocity distribution of the calculation area showed different at different times. (2) When the blade was in the state of small angle of attack, the flow around the blade was the attached flow. When it was greater than the critical angle of attack, there was a separate flow on the surface of the blade. The vorticity in the rotating area of the vertical axis wind turbine was large, and the value of vorticity decreased rapidly as it was far away from the rotating area. (3) During the rotation of the wind turbine, the total torque of the calculation model changed alternately with positive and negative as the angle of 
attack of the blades continuously changed.

\section{Acknowledgement}

This research was supported by Natural Science Foundation of China under Grant No. 51465010 \& Innovation Project of GUET Graduate Education No. 2017YJCX10.

\section{References}

1. X Lei. "Review of Wind Power Generation and Its Technology Development." Construction Engineering Techniques and Design( 2017)

2. S Liang, Q. Y. Ru, H. W. Jin. "Calculation of Starting Performance of Vertical Axis Wind Turbine Using Dynamic Grid and Sliding Grid Technology." Renewable Energy(2016)

3. J. W. Bin. "Research on aerodynamics of H-type vertical axis wind turbine." Chongqing University(2016)

4. S. C. Guang, Z. Yuan, W. G. Qing, et al. "Influence of inflow velocity and airfoil thickness on aerodynamic performance of vertical axis wind turbines." Academic Conference of Jiangsu Engineering Thermophysics Society(2014)

5. H. Y. Wu, L. G. Jian, Y. Ke. "Aerodynamic characteristics of low Reynolds number airfoil of S809 wind turbine under wingtip vortex effect." Chinese Journal of Solar Energy(2016)

6. M. Yi. "Research on flow field simulation and airfoil design method for vertical axis wind turbine under variable speed ratio." Harbin Institute of Technology(2015)

7. Z. S. Shuai. "Computational Fluid Dynamics and Its Applications." Huazhong University of Science and Technology Press(2011)

8. W. F. Jun. "Computational Fluid Dynamics Analysis: Principles and Applications of CFD Software." Thermal Power Engineering(2005) 